\title{
Clínica da ansiedade: aplicativo Android para educação em saúde mental
}

\author{
Anxiety clinic: Android app for mental health education \\ Clínica de ansiedad: aplicación de Android para educación en salud mental
}

Recebido: 20/08/2021 | Revisado: 26/08/2021 | Aceito: 30/08/2021 | Publicado: 01/09/2021

\author{
Adelma do Socorro Gonçalves Pimentel \\ ORCID: https://orcid.org/0000-0003-0048-4976 \\ Universidade Federal do Pará, Brasil \\ E-mail: adelmapi@ufpa.br \\ Júlio Ricardo Gomes Mota \\ ORCID: https://orcid.org/0000-0002-2291-9718 \\ Serviço Nacional de Aprendizagem Comercial, Brasil \\ E-mail: julioorg@gmail.com
}

\begin{abstract}
Resumo
Apresentamos o aplicativo Clinica da Ansiedade criado para esclarecer as formas de manifestação psicológica e psicopatológica. O aplicativo está disponível gratuitamente na playstore, sistema Android para uso como um recurso de intervenção no manejo clinico. A relevância do aplicativo se deve a elevada incidência mundial da ansiedade. Utilizou-se a linguagem de programação Kotlin, recomendada pelo Google. O aplicativo é composto por dez telas de navegação simples com diálogo entre textos e imagens coloridas em todas as telas. Aborda: fundamentação fenomenológica; imersão, imaginação; surgimento da ansiedade, pressões sociais; Covid-19; Desamparo; Dinâmica da ansiedade; A pessoa fora da caixa; sintomas físicos, psicológicos e sociais; Sistema multiaxial DSM-IV e o categorial-dimensional, DSM-V; sistemas científicos não podem ser camisas de força a serviço do Psiquiatra e psicólogo; Superar o Medo; e Homeostase. O aplicativo possui uma base epistemológica fenomenológica e gestáltica. Ao oferecê-lo propomos um material para diálogo do cliente internauta com os profissionais de saúde, visando compreender a dinâmica existencial da sua ansiedade e realizar tratamento clinico.
\end{abstract}

Palavras-chave: Ansiedade; Aplicativo; Android; Educação; Saúde.

\begin{abstract}
We present the application anxiety clinic created to clarify the forms of psychological and psychopathological manifestations. The application is available for free on the playstore, Android system for use as an intervention resource in clinical management. The app's relevance is due to the high incidence of anxiety worldwide. The Kotlin programming language recommended by Google was used. The application consists of ten simple navigation screens with dialog between texts and colored images on all screens. Approaches: phenomenological foundation; immersion, imagination; emergence of anxiety, social pressures; Covid-19; Helplessness; Dynamics of anxiety; The person outside the box; physical, psychological and social symptoms; Multiaxial system DSM-IV and the categoricaldimensional system, DSM-V; scientific systems cannot be straitjackets in the service of the Psychiatrist and psychologist; Overcome Fear; and Homeostasis. The app has a phenomenological and gestalt epistemological basis. By offering it, we propose a material for the Internet client to dialogue with health professionals, in order to understand the existential dynamics of their anxiety and carry out clinical treatment.
\end{abstract}

Keywords: Anxiety; Application; Android; Education; Health.

\begin{abstract}
Resumen
Presentamos la aplicación Clinica da Ansiedade creada para aclarar las formas de manifestaciones psicológicas y psicopatológicas. La aplicación está disponible de forma gratuita en PlayStore, sistema Android para su uso como recurso de intervención en la gestión clínica. La relevancia de la aplicación se debe a la alta incidencia de ansiedad en todo el mundo. Se utilizó el lenguaje de programación Kotlin recomendado por Google. La aplicación consta de diez pantallas de navegación sencillas con diálogo entre textos e imágenes en color en todas las pantallas. Enfoques: fundamento fenomenológico; inmersión, imaginación; aparición de ansiedad, presiones sociales; COVID-19; Impotencia; Dinámica de la ansiedad; La persona fuera de la caja; síntomas físicos, psicológicos y sociales; El sistema multiaxial DSM-IV y el sistema categórico-dimensional, DSM-V; los sistemas científicos no pueden ser camisas de fuerza al servicio del Psiquiatra y del psicólogo; Superar el miedo; y homeostasis. La aplicación tiene una base epistemológica fenomenológica y gestáltica. Al ofrecerlo, proponemos un material para que el cliente de Internet dialogue con los profesionales de la salud, con el fin de comprender la dinámica existencial de su ansiedad y realizar un tratamiento clínico.
\end{abstract}

Palabras clave: Ansiedad; Aplicación; Androide; Educación; Salud. 


\section{Introdução}

Neste texto apresentamos o aplicativo em Psicologia Clínica criado pelos autores para contribuir para o manejo clínico da ansiedade; e com o esclarecimento a sociedade brasileira, em toda a sua amplitude, sobre o que é a ansiedade e suas formas de manifestação psicológica e psicopatológica. O trabalho se vincula aos projetos da linha de pesquisa Fenomenologia: teoria e clínica do programa de pós-graduação em Psicologia da UFPA, no escopo da Educação para a Saúde Mental, em que nos valemos para delinear a estrutura de conteúdo, de uma linguagem clara e comunicativa para o manejo clínico do tratamento de adultos, entre 20 e 60 anos, atendidos pela equipe de Psicólogos e Psiquiatra coordenados pela primeira autora, em uma instituição pública de saúde mental no Pará/Região Norte.

Foi construído pelo segundo autor com aporte financeiro do PROAP/CAPES, usando na programação a linguagem Kotlin, compondo-se uma metodologia cujas etapas foram: análise, concepção, desenvolvimento, implementação e avaliação do design instrucional e tecnológico. O aplicativo está disponível gratuitamente na playstore, no link https://play.google.com/store/apps/details?id=com.app.clinica_gestaltica_ansiedade, loja de aplicativos virtuais para o sistema Android para ser usado por internautas adultos, e por profissionais de saúde, com destaque aos Psicólogos como um dos recursos de intervenção no manejo clinico da ansiedade.

A relevância social e cientifica da elaboração do aplicativo se deve a elevada incidência mundial da ansiedade, bem como sua presença na vida de toda a população, pois é considerada um "Conjunto complexo de emoções formado pelo medo, culpa, amargura e vergonha, sendo o medo a emoção dominante (Serra, 1980). Além da forma comum presente em nosso cotidiano, a ansiedade se manifesta no modo de transtornos mentais que podem ser classificados em: transtornos do pânico, de ansiedade generalizada, fobias específicas e transtornos de ansiedade social.

Ressaltamos que, no ano de 2020 a pandemia da COVID-19 alterou a percepção das pessoas sobre suas emoções, ou seja, houve uma intensificação da ansiedade, até então genericamente vivenciada como algo comum. Naquele cotidiano pandêmico tornou-se um sofrimento recorrente, associada a indefinição do curso da doença; a imprevisibilidade do controle das formas graves; e às condições de isolamento e quarentena impostas pelas agencias reguladoras federais, estaduais e municipais. Em 2021, o conhecimento sobre a mutação, infecção, sintomatologia do Corona vírus e a aplicação massiva no mundo das vacinas contribui para melhorar o ânimo e a disposição em saúde mental das pessoas, especificamente no que se refere a ansiedade. (Pimentel \& Malcher, 2020)

Etimologicamente, a palavra ansiedade é oriunda do grego "Agkho, significando estrangular, sufocar, oprimir" (Pereira, 1997). Os correlatos são: angústia, do latim "angustia, derivado do grego Agkho; pânico, do grego Panikon, relacionado ao Deus mitologico Pã, (Viana, 2010, p18). A autora apontou que o humor alterado de Pã foi associado à sua "feiura"1 e ao abandono materno. Seus gritos noturnos impunham medo. Do ponto de vista psiquiátrico, a ansiedade é alocada entre os transtornos mais frequentes na população geral (Schatzberg; Debattista, 2017). Segundo Moura et al. (2018) é avaliada como uma resposta natural do corpo frente a um estímulo que é indispensável para a autopreservação, levando o indivíduo para o confronto da situação, agindo com impulso e motivação a fim de preservá-lo. Porém, é entendida como patologia, caso ela se manifeste de forma exacerbada e de prolongada duração sendo desproporcional ao estímulo ansiogênico, afetando a vida diária do indivíduo e impossibilitando sua capacidade de adaptação (Claudino \& Cordeiro, 2016).

Situando a grande incidência da ansiedade na vida das pessoas estamos pesquisando e desenvolvendo estratégias de intervenção na clínica psicológica fenomenológica e gestáltica; portanto, elaborar um aplicativo visa informar e esclarecer a sociedade que a vivencia da ansiedade requer sair do contexto do silenciamento e do preconceito; e aos profissionais de saúde

\footnotetext{
${ }^{1}$ A ideia de feiura deriva de uma imposição estética da indústria da beleza, que desconsidera a condição subjetiva da percepção do belo que implica encantar-se pelo outro, para além do modelo padronizado.
} 
que é necessário desconstruir a lógica da patologização, em que a ansiedade é definida como um distúrbio mental, e a medicalização como uma ferramenta "indispensável" ao tratamento clínico da mesma. Ponderamos que é necessário conhecer as dinâmicas sociais envolvidas na gênese da ansiedade, bem como o modo de funcionamento psíquico singular, ou seja, a vivencia da ansiedade. Assim, é possível que a pessoa que vivencia a condição ansiosa se envolva e responsabilize pela promoção de seu tratamento interdisciplinar. (Carvalho \& Costa, 2020; L \& Barbosa, 2019)

De acordo com Barra, Paim, Dal Sasso, Colla (2017, p 2)

É possível constatar uma proliferação de tecnologias e aplicativos móveis (m-saúde/m-health) que estão colaborando para a construção de uma nova modalidade de assistência em saúde, no qual as informações referentes à saúde das pessoas se fazem oportunas. Diversos estudos apontam que tais aplicativos podem ser utilizados para otimização dos resultados e redução dos riscos em saúde, bem como, para compreensão dos fatores determinantes que promovem a saúde e/ou que levam à doença.

Por sua vez, Filho, Machado e Silva (2019) verificaram vinte e quatro aplicativos gratuitos, em português "Para smartphones até outubro de 2017, sistemas operacionais Android (Google Play) e iOS (App Store) que pudessem contribuir para o auxílio de psicoterapeutas na evolução clínica de seus clientes." $\mathrm{Na}$ análise encontraram os aplicativos voltados para a ansiedade: Ansiedade - Controle do Humor e Chat, desenvolvido pela MOB Aplicativos; Controle de Ansiedade de João Luz; Diário - Controle do Humor de Daylio; Querida Ansiedade, de Terapia a Distância. Os autores apontam que os aplicativos foram categorizados "6 grupos: Relaxamento, Autoconhecimento, Jogos Dinâmicos, Psicoeducação e Bate-papo", (p.8). além disso, os aplicativos dispõem de textos possuem áudios, vídeos e dicas sobre uma saúde mental mais saudável ou como reverter quadros de ansiedade." (p.11).

Bruno et.al. (2021) levantaram aplicativos associados aos conceitos de bem-estar, fitness e ao uso pelo internauta, sem necessitar diretamente da mediação do Psicoterapeuta: Psicólogo/Psiquiatra. Informam que a produção de aplicativos aumentou em 2020 ante a pandemia da Covid-19; entretanto, a Apple havia definido o ano de 2018 "como a grande tendência" de aplicativos no campo do 'autocuidado' (self-care) (p.35). Os autores afirmam que no campo dos aplicativos foi criado um Ecossistema digital combinado por corporações de tecnologia, ciência e sociedade constituído por algorítmicos de captura, análise e uso de informações psíquicas e emocionais extraídas de volumes de dados, compondo uma Economia Psíquica dos Algoritmos:

Os PsiAps abrangem um escopo amplo e heterogêneo de serviços e funcionalidades relacionadas à saúde mental: vão desde técnicas de meditação, passando por controle do sono ou de outras funções emocionais e fisiológicas, até a realização de testes psicológicos e terapia guiada. Têm o potencial de oferecer aos usuários informações e serviços, acesso a dados em tempo real, orientações sobre condições clínicas e incentivo a hábitos benéficos à saúde psíquica, emocional e física. Ampliam a coleta de informações psicológicas e emocionais, bem como as predições que se pretendem extrair dessas coletas para fins comerciais, a pesquisa médica, clínica e cientifica (p.35)

Por fim, Souza e Lima (2020) criticam a orientação descomedida da vida que alguns internautas associam aos paradigmas tecnológicos de informação e comunicação aplicados as interações virtuais. Os autores apontam a regulamentação imposta pelas redes sociais virtuais como uma das formas de maximização dos paradigmas. Em nossa compreensão precisamos desvelar os limites entre uso exagerado da internet e torna-la fonte de sofrimento psíquico.

Quando os profissionais de saúde: Psicóloga clínica e Psiquiatra conhecem a necessária reflexão sobre coleta de dados e funções dos algoritmos de controle da comunicação dos usuários da internet e redes sociais virtuais, podem contribuir para compor soluções em educação para saúde no âmbito da produção de aplicativos que não sejam norteados pelos princípios apontados em Souza e Lima (2000) no uso das redes sociais: não sentir-se só; sensação de ser escutado; ilusão de escolha autônoma da atenção aos conteúdos virtuais. 


\section{Metodologia}

O aplicativo Clínica da Ansiedade é um material produzido para orientar pessoas a desconstruir a concepção de ansiedade, unicamente associada a uma premissa patológica, para situa-la no horizonte da vivencia e da autocompreensão das causas que a provocam. É focado na plataforma Android, já que, de acordo com a International Data Corporation IDC-Brasil, o país, atualmente possui 83,8\% do mercado de smartphones. Para Ortiz (20021), o mercado de TIC no Brasil deve crescer 7\% em 2021, mesmo sob o impacto da pandemia. A estimativa é da IDC Brasil e faz parte do estudo IDC Predictions que antecipa as tendências e movimentos de mercado para os 110 países em que atua, como ilustra o Gráfico 1:

Gráfico 1. Projeção de uso.

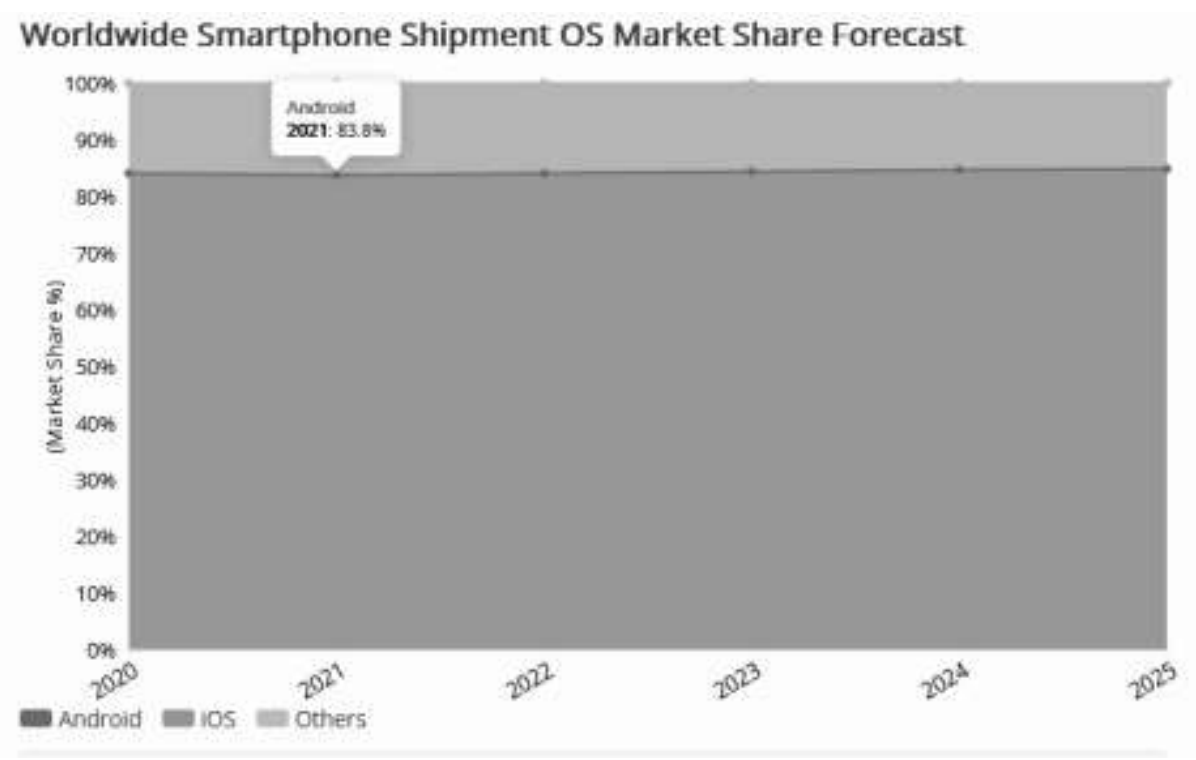

Fonte: https://www.ide.com/promo/smartphone-market-share

Na pesquisa realizada pela Bain e Company, 97\% dos usuários do Brasil utilizam o smartphone para acessar a internet, sendo que para $83 \%$ desses usuários é o único meio de acesso. O Gráfico 2, a seguir evidencia a importância do acesso via smartphone.

Gráfico 2. Acesso à internet por smartphone.
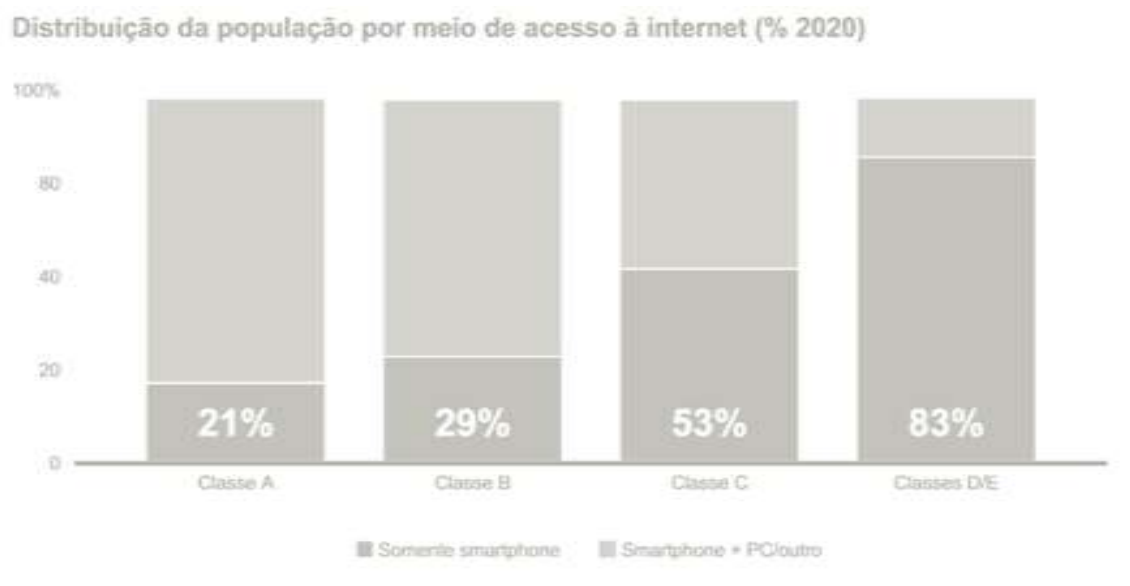
Para o desenvolvimento do projeto foi utilizada a linguagem de programação Kotlin, que é recomendada pelo Google desde 2018, sendo a principal linguagem de programação para desenvolvimento de aplicativos Android. A estrutura do aplicativo é composta por um menu com dez telas de navegação simples e fácil. Há um diálogo entre textos e imagens coloridas em todas as telas para deixar atraente o convite ao uso do aplicativo. As telas são, como mostra a Figura 1: 1) Introdução que descreve a fundamentação fenomenológica do aplicativo; 2) Imersão, um convite a adentrar no conteúdo e a usar a imaginação; 3) Como surge a ansiedade, abordagem das pressões sociais e um convite a pensar a vivencia; Covid-19; 4) O que é desamparo; 5) Dinâmica da ansiedade, mencionando experiencias traumáticas; 6) A pessoa fora da caixa, um panorama sobre sintomas físicos, psicológicos e sociais; 7) O sistema multiaxial DSM-IV e o categorial-dimensional, DSM-V, como sistemas de classificação que não podem ser considerados camisas de força da pessoa; 8) Uso de manuais: sistemas científicos que muitas vezes servem ao Psiquiatra e ao psicólogo não a pessoa que sofre com ansiedade; 9) Superar o Medo; 10) Homeostase:

Figura 1. Telas do app.

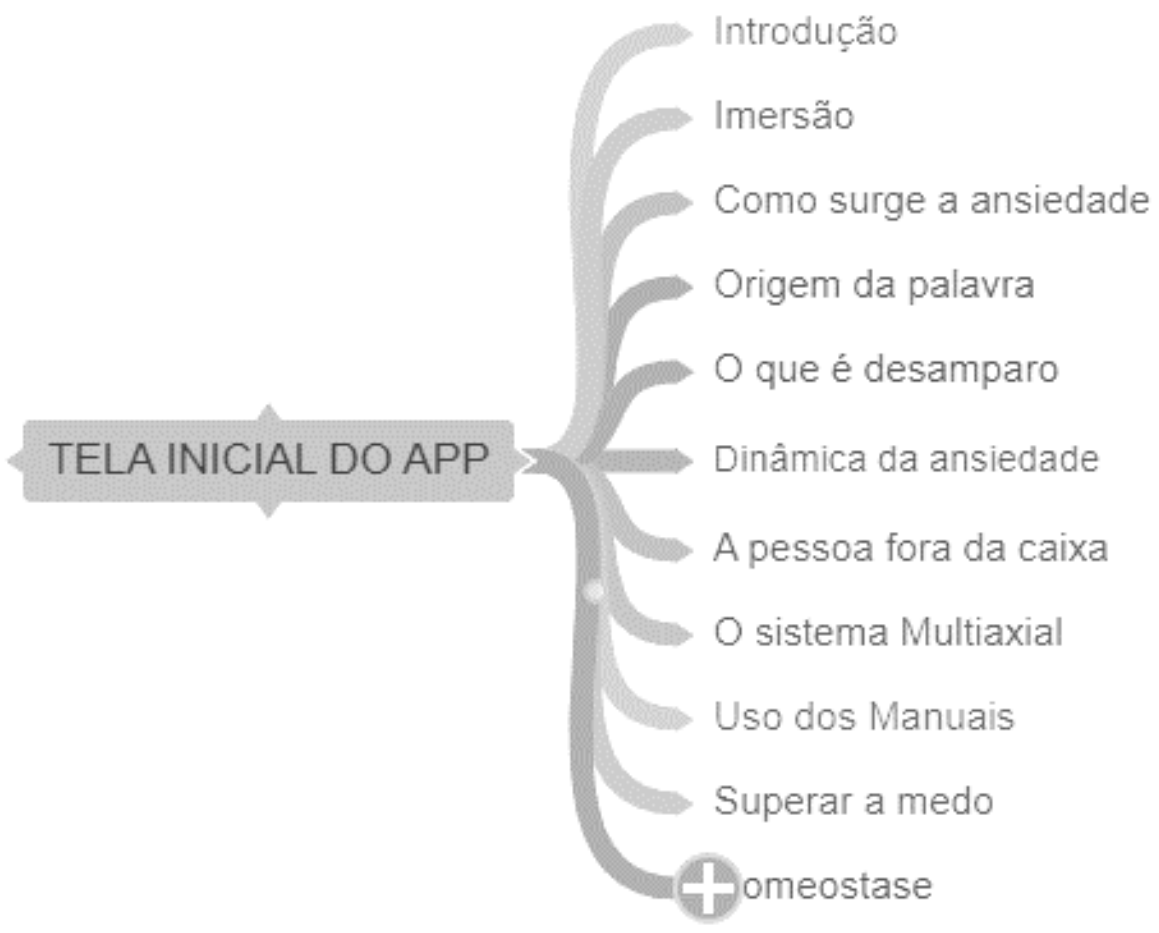

Fonte: Pesquisa (2021).

O Aplicativo é constituído em uma série de páginas contendo conteúdo de imagem e texto que discorrem sobre o tema ansiedade. O mapa mental a seguir ilustra a organização do conteúdo presente no aplicativo. As dez páginas podem ser acessadas por dois mecanismos de navegação: um menu drawer que permite que o usuário navegue por meio de uma gaveta de opções, similar a um sumário; e um теnи na parte inferior que permite passar para a próxima página. 
Figura 2. Menus.

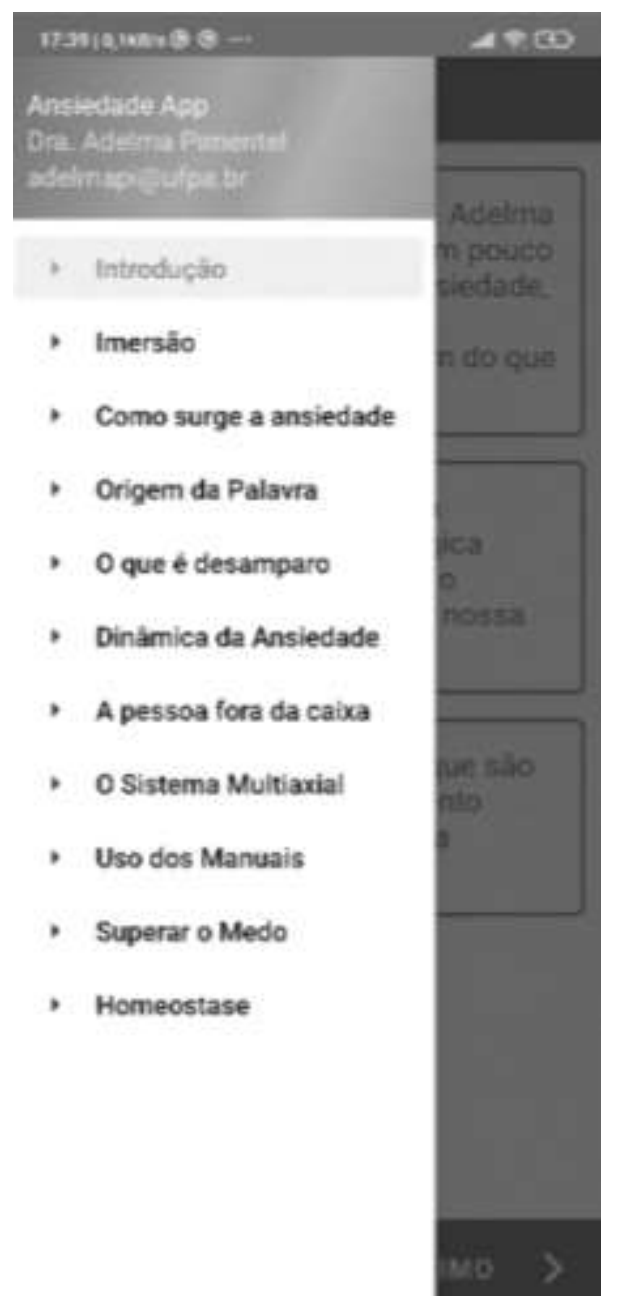

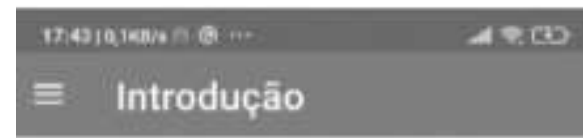

Olà Eu sou a professora Dra. Adelma Pimentel, vamos entender um pouco mais sobre a vivéncia da Ansiedade, uma situaçẵo que acontece conosco, sendo mais comum do que vocé pensa, ou sabe.

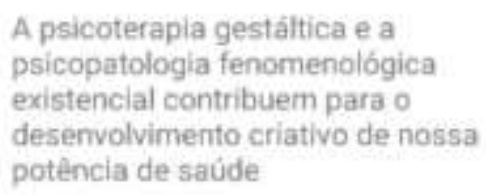

A psicoterapia gestáltica e a psicopatologia fenomenológica existencial contribuem para o desenvolvimento criativo de nossa potencia de saúde

E importante compreender que são várias as formas de tratamento da ansiedade. De preferència interdisciplinat.

Fonte: Autores.

\section{Considerações Finais}

Ressaltamos que nosso trabalho não se alinha a tendência inserida na lógica do Ecossistema digital composto pela indústria das Tecnologias de Informação e Comunicação que elabora algorítmicos de captura, análise e uso de informações psíquicas e emocionais extraídas de volumes de dados, compondo uma Economia Psíquica dos Algoritmos. Em nosso afazer há uma base epistemológica presente no aplicativo para que os profissionais de saúde, quando navegarem pelos menus digitais observem a linguagem fenomenológica e gestaltica. Há ainda o acréscimo de uma crítica a medicalização e a classificação indiscriminada às patologias mentais. Consideramos que é necessário desenvolver escuta empática em saude mental associada à oferta aos internautas-clientes dos serviços de Psicologia Clínica. A ética do cuidar é base para que a produção de estratégias, que se vale de meios digitais e das Tecnologias de Informação e Comunicação, seja mediada pela orientação do Psicólogo, do Psiquiatra, e da equipe de saúde multidisciplinar, conforme a demanda clínica do cliente. Assim, ao oferecer o aplicativo Clínica da Ansiedade nossa proposta é que o material seja um passo no diálogo do cliente internauta com a equipe de saúde, visando compreender a dinâmica existencial da vivencia da sua ansiedade e realizar tratamento clinico. 


\section{Agradecimentos}

Ao professor Dr Leandro Passarinho, Vice coordenador do programa de pós-graduação em psicologia da UFPA pela gestão equitativa dos recursos da CAPES/PROAP.

\section{Referências}

Barra, D. C. C.; Paim, S. M. S.; Dal Sasso, G. T. M.; Colla, G. W. (2017) Texto Contexto Enferm, 26(4). Métodos para desenvolvimento de aplicativos móveis em saúde: revisão integrativa da literatura.

Bruno, F. G., et al. (2021). Tudo por conta própria: autonomia individual e mediação técnica em aplicativos de autocuidado psicológico. RECIS - Revista Eletrônica de Comunicação, Informação e Inovação em Saúde, 15(1), 33-54.

Carvalho Júnior, G., \& Costa, A. (2020). Plenitude: Aplicativo mobile para melhorar a qualidade de vida de pessoas com transtornos depressivos e ansiedade. In Anais da VIII Escola Regional de Computação do Ceará, Maranhão e Piauí, (pp. 268-275). SBC. 10.5753/ercemapi.2020.11494

Claudino, J., \& Cordeiro, R. (2016). Níveis de ansiedade e depressão nos alunos do curso de licenciatura em enfermagem. O caso particular dos alunos da Escola Superior de Saúde de Portalegre. Millenium-Journal of Education, Technologies, and Health. (32):197-210.

Filho, J. P. M. V., Machado, E. T. de M., \& Silva, A. N. (2019). Análise dos aplicativos para smartphones com potencial terapêutico em psicoterapia disponíveis em língua portuguesa. Revista Cearense de Psicologia. 1-15

L., Paula, \& Barbosa, J. (2019). LifeReview: Um modelo para acompanhamento de pessoas com transtorno de ansiedade. In Anais do XV Simpósio Brasileiro de Sistemas de Informação, (pp. 56-62). SBC.

Moura, I. M., Rocha, V. H. C., Bergamini, G. B., Samuelsson, E., Joner, C., Schneider, L. F., \& Menz, P. R. (2018). A terapia cognitivo-comportamental no tratamento do transtorno de ansiedade generalizada. Revista Científica Da Faculdade De Educação E Meio Ambiente, 9(1), 423-441. https://doi.org/10.31072/rcf.v9i1.557

Ortiz, S. M, (2021) previsões da idc brasil para 2021 apontam que mercado de tic crescerá 7\%. https://www.idc.com/getdoc.jsp?containerid=prla47452221

Pimentel, A., \& Malcher, M. de N. (2020). Emoções à flor da pele: riscos à saúde psíquica em tempos de coronavírus, In, COVID-19: Saúde da mente e do corpo. Elói Martins Senhoras (organizador). Universidade Federal de Roraima (UFRR)

Pimentel, A., \& Gomes, J. (2021). Aplicativo Clinica da Ansiedade. Disponível na playstore, link https://play.google.com/store/apps/detai 1s?id=com. app.ansiedade $=$ pt-BR

Schatzberg, A. F., \& Debattista, C. (2017). Manual de Psicofarmacologia Clínica. (8a ed.), Artmed. Ed. p 2-14.

Serra, A. S. V. (1980). O que é ansiedade? Psiquiatria clássica, 1(2), 93-104.

Souza, R. W. V. de, \& Lima, G. M. M. de. (2020). A Vida Conforme O Paradigma Tecnológico: Violência e solidão no mundo globalizado. Revista Científica do UniRios 2020.1|380-387.

Viana, M. de B. (2010). Mudanças nos conceitos de ansiedade nos Séc. XIX e XX: da "Angstneurose” ao DSM-IV. São carlos. Tese de Doutorado. 